\title{
Correction to: Femoral and tibial bone bruise volume is not correlated with ALL injury or rotational instability in patients with ACL-deficient knee
}

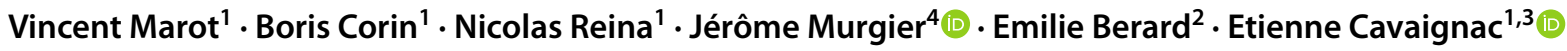

Published online: 28 May 2020

(c) European Society of Sports Traumatology, Knee Surgery, Arthroscopy (ESSKA) 2020

\section{Correction to: \\ Knee Surgery, Sports Traumatology, Arthroscopy \\ https://doi.org/10.1007/s00167-020-06045-x}

The affiliation of author Jérôme Murgier was published incorrectly in the original article. His correct affiliation is:

Aguiléra Private Clinic, Ramsey Générale de Santé, Biarritz, France

ORCID ID: https://orcid.org/0000-0002-9237-1372

Publisher's Note Springer Nature remains neutral with regard to jurisdictional claims in published maps and institutional affiliations.

The original article can be found online at https://doi.org/10.1007/ s00167-020-06045-x.

Etienne Cavaignac

cavaignac.etienne@gmail.com

1 Department of Orthopedic Surgery and Trauma, Pierre Paul Riquet University Hospital, Toulouse, France

2 Department of Epidemiology, Health Economics and Public Health, UMR1027 INSERM-University of Toulouse III, Toulouse University Hospital, Toulouse, France

3 Department of Orthopedic Surgery, Hôpital Pierre Paul Riquet, CHU Toulouse, Rue Jean Dausset, 3105 Toulouse, France

4 Aguiléra Private Clinic, Ramsey Générale de Santé, Biarritz, France 